\title{
Psychological impact and coping strategies of medical students during university closure due to COVID-19 in a state university in Sri Lanka
}

Y M Rohanachandra, P S Alles, L Amarakoon, S Prathapan

\section{Abstract \\ Introduction}

Medical students are a vulnerable group to develop mental health problems due to high academic pressure, competitive training, and financial restraints. Loss of routine, disengagement from peers and adapting to distant learning during the pandemic may worsen psychological distress in medical students.

\section{Aims}

To identify the psychological impact and coping strategies of medical students during the pandemic.

\section{Methods}

The study instruments were emailed as a Google form to all medical students of the University of Sri Jayewardenepura, Sri Lanka. A specifically designed questionnaire was used to gather the sociodemographic details and assess coping skills. The Depression Anxiety and Stress Scale (DASS-21) was used to measure the psychological impact.
Results

A total of 527 students responded. The main worry among the students was upcoming examinations (74.4\%). Depressive symptoms were present in $40.8 \%$, anxiety in $34 \%$ and high levels of stress were seen in $24.7 \%$. Depression $(p<0.01)$, anxiety $(p<0.05)$ and stress $(p<0.01)$ were significantly higher in students with a past history of psychiatric disorders. The main coping strategy of the participants was engaging more with family (73.4\%). Half of the students $(50.7 \%)$ reported participating in all distant teaching sessions. Help seeking behaviour among students appeared to be poor, as only $40.5 \%$ of students provided their contact details and consented to be contacted by the investigators to provide support.

\section{Conclusions}

Given the high levels of psychological distress, supportive strategies should be designed to minimize the psychological impact in this vulnerable group.

Key words: depression, anxiety, stress, university students, pandemic

SL J Psychiatry 2021; 12(2): 4-9

\section{Introduction}

On $30^{\text {th }}$ January 2020, the World Health Organization (WHO) determined that the outbreak of coronavirus disease (COVID-19) constituted a Public Health Emergency of International Concern (1). In an effort to prevent the spread of COVID-19, the WHO recommended stopping activities that could potentially lead to spread of the illness. In keeping with this, the government of Sri Lanka closed universities on the $13^{\text {th }}$ of March 2020, and with this, all clinical rotations, lectures, small group discussions and practical sessions of medical students were temporarily suspended. Distant learning methods were employed to conduct lectures, tutorials and small group discussions.

Highly competitive training, academic pressure, exposure to patients in clinical settings, financial constraints and lack of sleep have been reported as factors that may contribute to medical students being an especially vulnerable group to development of mental health problems (2, 3). A meta-analysis report that the global prevalence of depression among medical students to be 
as high as $28.0 \%$ (4). Medical students are also at a higher risk of suicidal ideation, burnout, substance abuse and stress (5). Studies done on psychological distress among medical students in Sri Lanka have demonstrated the distress rates in students in Sri Lanka to be higher than in other countries (6).

With the closure of universities, the resulting loss of routines, structure and peer support networks may add to the psychological distress in medical students. Adapting to distant learning methods could also lead to additional psychological distress, especially in lower middle income countries (LMIC), due to difficulty in accessing online teaching as a result of narrow broadband widths, unreliable internet connectivity and due to many students only having basic smartphones to access the online learning content $(7,8)$.

Many studies have focused on the mental health of health care workers during the pandemic (9-19). However, only few studies have focused on the psychological impact of the pandemic on medical students (20-25). To our knowledge, no such studies have been carried out in Sri Lanka. Therefore, this study aimed at assessing the prevalence of psychological distress and coping strategies in medical students in Sri Lanka and the associated factors.

\section{Methods}

This study was carried out among medical students from the first to the final year of the University of Sri Jayawardenapura, Sri Lanka. The email addresses of the students were obtained through the administration of the University. All students who were enrolled at the medical faculty at the time of the study, were invited to participate. The study instruments were emailed as a Google form. Coercion was minimal, as the study was by invitation and the participants was anonymous.

A specifically designed questionnaire was used to collect socio-demographic details, coping strategies and associated risk factors for development of psychological consequences. The validated English version of the 21- item Depression Anxiety and Stress Scale (DASS-21) was used to assess psychological impact (26). The Depression, Anxiety and Stress Scale - 21 (DASS-21) consists of 3 self-report scales designed to measure states of depression, anxiety and stress. Each of the three scales contains 7 items, divided into subscales with similar content. Scores for depression, anxiety and stress are calculated by summing the scores for the relevant items and multiplying by two (26). The Chi square test was used to analyze categorical data, t test and ANOVA were used to identify difference in means between groups. Ethical clearance and, permission to conduct the study was obtained from the Faculty of Medical Sciences, University of Sri Jayawardenapura.

\section{Results}

\section{Socio-demographic details}

The questionnaire was emailed to 939 students with a response rate of $56.1 \%(n=527)$. Seventy percent of respondents $(n=369)$ were female. The mean age of the participants was 23.48 years (SD+/- 2.087) and most (88.6\%, n=467) were Sinhalese. The highest number of respondents $(30 \%, n=158)$ were students in their first year of study. Of them, $5.9 \%(n=31)$ had a past history of psychiatric disorders.

\section{Psychological impact}

The main worries among the medical students were about upcoming examinations $(74.4 \%, n=389)$, taking a longer time than expected to complete their undergraduate career $(68.1 \%, \mathrm{n}=356)$ and worries about contracting COVID-19 (45.7\%, $n=239)$. Of the participants, $15.1 \%(n=79)$ were concerned about difficulties in participating in distant learning.

Of the total, $68.7 \%(n=362)$ of the respondents experienced difficulties in working up the initiative to engage in daily activities, $62.6 \%(n=330)$ had the tendency to overreact to situations, $57.3 \%(n=302)$ found it difficult to relax and $56.7 \%(n=299)$ were unable to experience any positive feeling at all.

Of the total participants, $40.8 \%$ had depressive symptoms, 34\% had anxiety and $24.7 \%$ had high levels of stress (Table 1). Depression ( $<<0.01)$, anxiety $(\mathrm{p}<0.05)$ and stress $(\mathrm{p}<0.01)$ were significantly higher in students with a past history of psychiatric disorders. Depressive symptoms were also significantly higher in first and second years compared to fourth year $(\mathrm{p}<0.05)$. Depression, anxiety or stress was not associated with the gender, ethnicity, family income or living circumstances (Table 2).

\section{Coping strategies}

The main reported coping strategies of participants during university closure was engaging more with family (73.4\%, $\mathrm{n}=384)$, watching television and movies (70\%, $\mathrm{n}=366)$, preparing for examinations $(66.5 \%, \mathrm{n}=348)$ and listening to music (64.4\%, $\mathrm{n}=337)$.

\section{Participation in distant learning}

Half of the students $(50.7 \%, n=267)$ reported that they participate in all distant teaching sessions, while another $41.7 \%(n=220)$ claimed to participate in most sessions. The reasons for missing online teaching sessions were difficulties in affording data $(8.2 \%, n=43)$, lack of motivation for distant learning $(7.2 \%, \mathrm{n}=38)$ and lack of internet facilities $(2.7 \%, \mathrm{n}=14)$. 
Table 1. Levels of depression, anxiety and stress among medical students

\begin{tabular}{|l|c|c|c|c|c|} 
& Mild & Moderate & Severe & $\begin{array}{c}\text { Extremely } \\
\text { severe }\end{array}$ & Total \\
\hline Depression & $12.5 \%$ & $17.5 \%$ & $4.0 \%$ & $6.8 \%$ & $40.8 \%$ \\
\hline Anxiety & $8.3 \%$ & $15.4 \%$ & $4.6 \%$ & $5.7 \%$ & $34.0 \%$ \\
\hline Stress & $9.1 \%$ & $7.8 \%$ & $6.1 \%$ & $1.7 \%$ & $24.7 \%$ \\
\hline
\end{tabular}

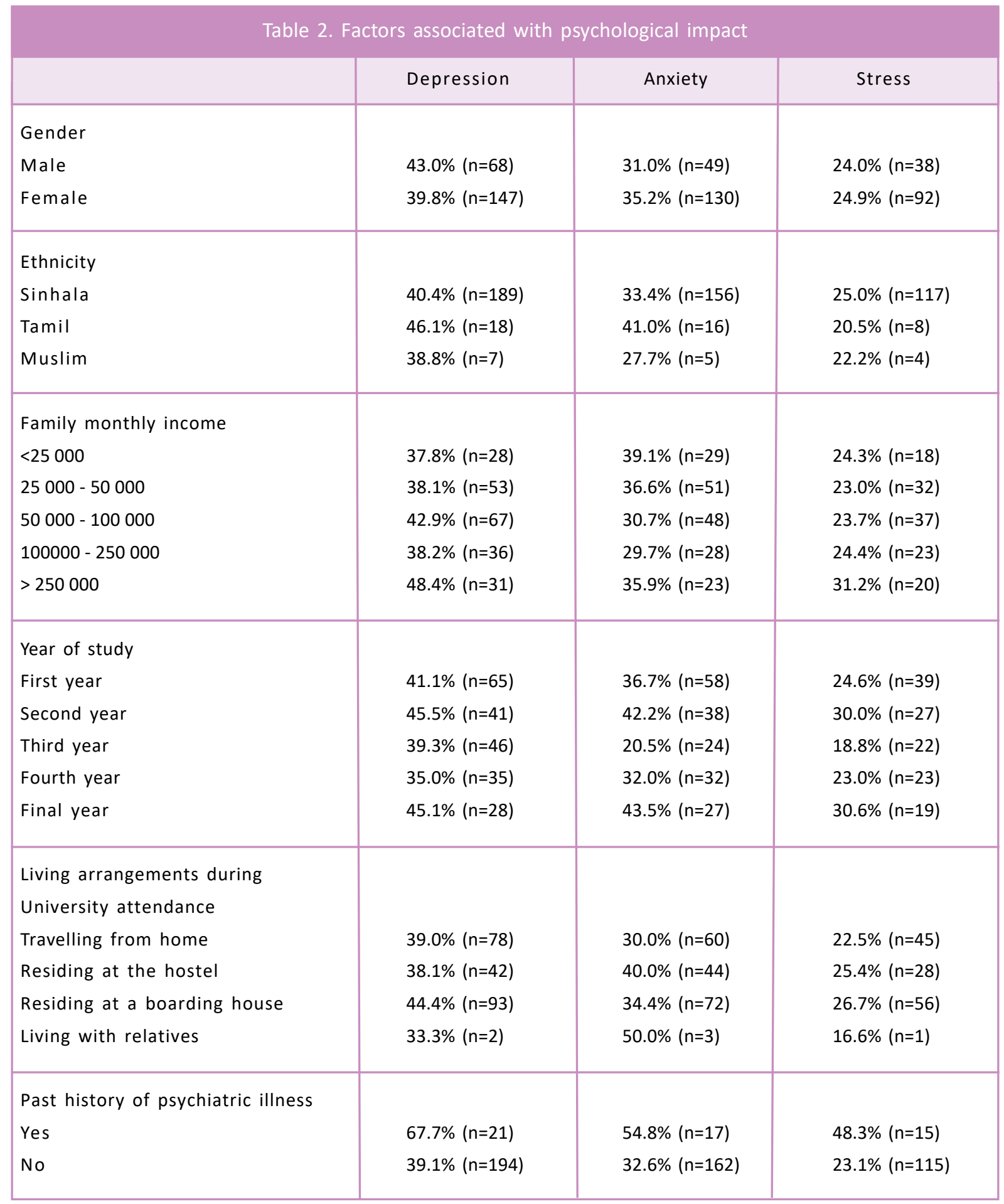




\section{Help seeking behaviour}

None of the participants contacted the investigators for psychological support despite the contact details of the investigators being provided, with an invitation to contact the investigators if they feel support is needed. Only $40.5 \%(n=214)$ of all students provided their contact details and consented to be contacted by the investigators to provide support if high levels of depression, anxiety or stress was detected.

\section{Discussion}

The findings of our study revealed that the major worries among medical students were about upcoming examinations (74.4\%) and about taking a longer time than expected to complete their undergraduate medical career (68.1\%). Less than half of the students (45.7\%) worried about contracting COVID-19, which was in contrast to the findings by Ahmed et al., who found that $75.8 \%$ of the students worried about getting infected with COVID19 (20). This may be explained by the fact that almost half of our sample (47.1\%) was first and second year students, who have not yet commenced clinical rotations, making them less likely to worry about contracting COVID-19.

Previous literature has shown the prevalence of depression among medical students during the current pandemic to range between $25 \%$ - 64\% (21-23). In our study, the prevalence of depressive symptoms was $40.8 \%$, which was comparable with these findings. The prevalence of anxiety in previous studies has ranged between $17 \%$ - 46\%, which was also comparable with the $34 \%$ found in our study (21-24). The findings on the relationship between gender and psychological distress among students are inconsistent. A study by Li et al., reported that female students tend to be more distressed during the current pandemic, while Liu et al. found no gender differences $(25,27)$. In keeping with the findings of Liu et al., our study did not reveal any gender differences with regards to anxiety, depression or stress.

Our findings show that $92.4 \%$ participated in all or most distant learning sessions, while only $2.5 \%$ participated in less than half of the distant learning sessions. In those who failed to participate in distant learning, the main reason was difficulty in affording data. Recognizing this difficulty, the universities took measures to create zoom accounts for all staff members through LEARN (Lanka Education and Research Network). This permitted staff to host zoom meetings which was free of charge for students. In addition, the recorded lectures were uploaded on to the Learning Management System (LMS), which could also be accessed free of charge for the students. However, these facilities were not available at the time of this study. Lack of motivation for distant learning was only found in $7.2 \%$ of participants. This is in contrast to previous studies, where approximately one third to half of the students reported poor motivation to engage in distant learning (28). Earlier studies have also described participation in distant learning as a major concern among medical students, but this only concerned a minority (15.1\%) of the students in the present study (29). The literature suggests that distant learning maybe especially difficult in low and middle income countries (LMIC) due to narrow broadband widths, lack of reliable internet connectivity, electricity supply and limited resources (e.g. laptops) $(7,8)$. Many of these factors also apply to Sri Lanka, which is also a LMIC. Despite these challenges, participation in distant learning was satisfactory in our sample of students. Factors that contributed to satisfactory participation and higher motivation for distant learning in this population should be further studied to identify strengths in the methods of distant learning used in this group of students, which maybe applicable to other LMIC.

Previous studies have shown that the main coping mechanism of medical students is maintaining online social interactions is through either social media or online chatting $(28,29)$. However, in our study, the main coping mechanism used was spending more time with family (70\%) and only about half of the students used social media as a coping mechanism. Lack of affordability of data, narrow broadband width and unreliable internet connection, especially in rural areas may all have contributed to social networking being less popular as a coping mechanism among Sri Lankan medical students. In addition, a majority of the medical students in Sri Lanka reside away from home during their university education and the lockdown may have provided them with opportunity to spend more time with their family, which may explain why spending time with family emerged as a more popular coping mechanism in these students.

Although high levels of psychological distress were found in our participants, only $40.5 \%$ of students provided their contact details and consented to be contacted by the investigators of the Department of Psychiatry to obtain support. This reluctance for help seeking has been widely described in previous literature. Stigma, fears about confidentiality, fear of social exclusion and lack of knowledge about the location of mental health services, among students, have been described as reasons for low help seeking among medical students (30). In the current setting, the students get minimal face to face contact with the staff of the Psychiatry Department until they start their clinical appointments in the third year. Therefore, unfamiliarity with the staff at the Psychiatry Department may also contribute to the low help-seeking behavior from study investigators, and students may have sought help from their assigned personal tutor instead.

The findings of this study also support previous recommendations that medical undergraduates should be screened for psychological disturbances and receive timely interventions during a pandemic (22). In addition, 
as the psychological distress was found to be much higher in students with a past history of mental health issues, arrangements should be made to closely monitor the mental health status of such students. Furthermore, incorporating discussions on how COVID-19 may affect mental health as a part of the distant learning may encourage help seeking behaviour in the students. Finally, providing information on how and where to obtain mental health services may also be useful, especially for first and second year pre-clinical students, who may be unfamiliar with mental health services. The Department of Psychiatry plays a key role in providing such information and should take and lead in designing appropriate psychological support services within the faculty. Staff in the Department of Psychiatry could offer to be freely available for in-person or online consultations for students and to refer to alternative mental health services when necessary. They can also take the lead in initiating discussions on impact of COVID-19 on mental health and encourage help-seeking behaviour of students.

\section{Limitations}

This was an online survey where the response rate was $56.1 \%$. The lack of affordability of data and lack of accessibility of internet facilities may have influenced the response rate and also students with higher psychological impact may not have responded due to lack of motivation. This may have affected the findings of this study.

\section{Conclusions}

High rates of psychological distress was detected among medical students during the pandemic. Therefore, supportive strategies need to be designed, focusing on the mental health needs of this population. Future research should aim to assess the baseline stress and mental health of medical students in order to compare the significance of the psychological distress associated with the pandemic.

\section{Acknowledgements}

None.

\section{Statement of Contribution}

All authors were responsible for the conception and design of the work. YR analyzed and interpreted the data and drafted the work. All authors revised it critically for important intellectual content, approved the final version to be published and agree to be accountable for all aspects of the work in ensuring that questions related to the accuracy or integrity of any part of the work are appropriately investigated and resolved.

\section{Declaration of interests}

There are no conflicts of interests

Y M Rohanachandra, P S Alles, L Amarakoon, Department of Psychiatry, Faculty of Medical Sciences, University of Sri Jayewardenepura, Sri Lanka

S Prathapan, Department of Community Medicine, Faculty of Medical Sciences, University of Sri Jayewardenepura, Sri Lanka

Corresponding author: Y M Rohanachandra

Email: yasodha_mk@yahoo.com, yasodha@sjp.ac.lk

http://orcid.org/0000-0002-3671-7674

\section{References}

1. Sohrabi C, Alzafi Z, O’Neill N, et al. World Health Organization declares global emergency: A review of the 2019 novel coronavirus (COVID-19). Int J Surg 2020; 76: 71-6.

2. Al Saadi T, Addeen SZ, Turk T, Abbas F, Alkhatib M. Psychological distress among medical students in conflicts: a cross-sectional study from Syria. BMC Med Educ. 2017; 17(1): 173.

3. Tian-Ci Quek T, Tam WS, X Tran B, et al. The global prevalence of anxiety among medical students: a metaanalysis. Int J Environ Res Public Health, 2019; 16(15): 2735.

4. Puthran R, Zhang MW, Tam WW, Ho RC. Prevalence of depression amongst medical students: A metaanalysis. Med Educ 2016; 50(4): 456-68.

5. Molodynski A, Lewis T, Khadum M, et al. Cultural variations in wellbeing, burnout and substance use amongst medical students in twelve countries. Int Rev Psychiatry 2020: 1-6.

6. Wimberly CE, Rajapakse H, Park LP, Price A, Proeschold-Bell RJ, Ostbye T. Mental well-being in Sri Lankan medical students: a cross-sectional study. Psychol Health Med 2020: 1-14.

7. Barteit S, Guzek D, Jahn A, Bärnighausen T, Jorge MM, Neuhann F. Evaluation of e-learning for medical education in low-and middle-income countries: A systematic review. Comput Educ 2020; 145: 103726.

8. Mittelmeier J, Rogaten J, Sachikonye M, Gunter A, Princeloo P, Rienties P. Understanding the adjustment of first-year distance education students in South Africa: Factors that impact students' experiences. Int Rev Res Open Dis, 2019; 20 (3). 
9. Spoorthy MS, Pratapa SK, Mahant S. Mental health problems faced by healthcare workers due to the COVID-19 pandemic - A review. Asian J Psychiatr. 2020; 51: 102119.

10. Lai J, Ma S, Wang Y, Cai Z, Hu J, Wei N, et al. Factors associated with mental health outcomes among health care workers exposed to coronavirus disease 2019 JAMA Netw Open. 2020; 3(3): e203976-e.

11. Babore A, Lombardi L, Viceconti ML, Pignataro S, Marino V, Crudele M, et al. Psychological effects of the COVID-2019 pandemic: Perceived stress and coping strategies among healthcare professionals. Psychiatry Res. 2020; 293: 113366.

12. Cai H, Tu B, Ma J, Chen L, Fu L, Jiang Y, et al. Psychological Impact and Coping Strategies of Frontline Medical Staff in Hunan Between January and March 2020 During the Outbreak of Coronavirus Disease 2019 (COVID-19) in Hubei, China. Med Sci Monit. 2020; 26: e924171-1.

13. Zhu Z, Xu S, Wang H, Liu Z, Wu J, Li G, et al. COVID19 in Wuhan: Sociodemographic characteristics and hospital support measures associated with the immediate psychological impact on healthcare workers. E Clinical Medicine. 2020; 24: 100443.

14. Kang L, Li Y, Hu S, Chen M, Yang C, Yang BX, et al. The mental health of medical workers in Wuhan, China dealing with the 2019 novel coronavirus. Lancet Psychiatry. 2020; 7(3): e14.

15. Zhang W-r, Wang K, Yin L, Zhao W-f, Xue Q, Peng M, et al. Mental health and psychosocial problems of medical health workers during the COVID-19 epidemic in China. Psychother Psychosom. 2020; 89(4): 242-50.

16. Liu C-Y, Yang Y-z, Zhang X-M, Xu X, Dou Q-L, Zhang $\mathrm{W}-\mathrm{W}$, et al. The prevalence and influencing factors in anxiety in medical workers fighting COVID-19 in China: a cross-sectional survey. Epidemiology \& Infection. 2020; 148: e98, 1-7.

17. Romero CS, Delgado C, Catalá J, Ferrer C, Errando C, Iftimi A, Benito A, De Andres J, Otero M. COVID-19 psychological impact in 3109 healthcare workers in Spain: The PSIMCOV group. Psychol Med. 2020; 14: 1-7.

18. Du J, Dong L, Wang T, Yuan C, Fu R, Zhang L, et al. Psychological symptoms among frontline healthcare workers during COVID-19 outbreak in Wuhan. Gen Hosp Psychiatry. 2020; 67: 144-45.

19. Shaukat N, Ali DM, Razzak J. Physical and mental health impacts of COVID-19 on healthcare workers: A scoping review. Int J Emerg Med. 2020; 13(1): 1-8.
20. Ahmed N, Khan A, Naveen HA, Moizuddin SM, Khan J. Concerns of Undergraduate Medical Students Towards an Outbreak of COVID-19. Int. J. Med. Res. Pharm. Sci. 2020; 6 (3): 5055-62.

21. Liu J, Zhu Q, Fan W, Makamure J, Zheng C, Wang J. Online Mental Health Survey in a Medical College in China During the COVID-19 Outbreak. Front Psychiatry, 2020; 11: 459.

22. Sartorao Filho CI, Rodrigues WC, de Castro RB, et al. Impact of Covid-19 Pandemic on Mental Health of Medical Students: A Cross-Sectional Study Using GAD7 And PHQ-9 Questionnaires. medRxiv, 2020.

23. Xiao H, Shu W, Li M, et al. Social Distancing among Medical Students during the 2019 Coronavirus Disease Pandemic in China: Disease Awareness, Anxiety Disorder, Depression, and Behavioral Activities. Int J Environ Res Public Health, 2020; 17(14): 5047.

24. Saddik B, Husseing A, Sharif-Askari F, et al. Increased levels of anxiety among medical and non-medical university students during the COVID-19 pandemic in the United Arab Emirates. medRxiv, 2020.

25. Li Y, Wang Y, Jiang J, et al. Psychological distress among health professional students during the COVID-19 outbreak. Psychol. Med., 2020: 1-3.

26. Rekha A. Adaptation and validation of the Depression, Anxiety, and Stress Scale (DASS21) among Students of the University of Colombo. 2012.

27. Liu X, J Liu, and X Zhong. Psychological State of College Students During COVID-19 Epidemic. Available at SSRN 3552814, 2020.

28. Arënliu A and Bërxulli D. Rapid assessment: Psychological distress among students in Kosovo during the COVID-19 pandemic.

29. Al-Tammemi A, Akour A and Alfalah L. Is it Just About Physical Health? An Internet-Based Cross-Sectional Study Exploring the Pyschological Impacts of COVID19 Pandemic on University Students in Jordan Using Kessler Psychological Distress Scale. Front. Psychol. 2020; 11: 562213.

30. Menon V, Sarkar S, Kumar S. Barriers to healthcare seeking among medical students: a cross sectional study from South India. Postgrad Med J. 2015; 91(1079): 477-82. 